\title{
Circadian rhythms of plasma cortisol in migraine
}

\author{
DEW E K . Z IEGLER, RUTH S. HAS A N E IN, \\ ALT A N KODANAZ, AND JOSEPH C. MEEK \\ From the Departments of Neurology, Biometry, and Medicine, Kansas University Medical Center, \\ College of Health Sciences and Hospital, Kansas City, Kansas, USA
}

S UM MARY Diurnal rhythm of plasma cortisol, of psychological state, and of pain was measured for two days in 25 migraine patients and eight control subjects. Fourteen of the migraine patients and none of the controls displayed either consistently high plasma cortisol or an occasional aberrant peak. Abnormal psychological findings, particularly depression, were found in the Minnesota Multiphasic Personality Inventory only in migraine patients with abnormal plasma cortisol levels. Neither psychological abnormality nor pain seemed the single cause of elevation of plasma cortisol.

Levels of plasma cortisol in man show a circadian rhythm, with a high point being recorded usually just before awakening and a progressive fall occurring during the waking hours and early hours of sleep (Yates and Urquhart, 1962). Studies on patients with pain have shown that plasma cortisol patterns deviate from the norm in some, but not all, such individuals. One study has concluded that the abnormal patterns of plasma cortisol levels are characteristic of patients with "organic" as opposed to "psychogenic" pain (Shenkin, 1964). There has been much evidence that plasma cortisol is altered profoundly by certain psychiatric states (Suwa et al., 1974; Carroll, 1976; Miyabo et al., 1976) but no studies have been done of the circadian rhythm of plasma cortisol in patients with painful conditions.

We thought it would be of interest to make a specific study of the circadian patterns of plasma cortisol in patients with migraine. The pain of migraine is frequently severe. Usually no organic lesion is found, yet the stereotyped nature of the pain pattern and the associated autonomic disturbances are persuasive evidence that the pain has a physiological base. On the other hand, the pain of migraine has unmistakable if variable associations with emotional stress and depression. It would, then, be difficult to predict whether the plasma cortisol patterns of migraine patients would resemble more those of patients with

Address for reprint requests: Dr D. K. Ziegler, Department of Neurology, University of Kansas Medical Center, Rainbow Boulevard at 39th, Kansas City, Kansas 66103, USA.

Accepted 2 February 1979 "organic," or those with "psychogenic" pain, and of particular interest to study the circadian pattern of the hormone.

We have studied the plasma cortisol levels for two and a half days and the presence (and degree) or absence of headache, and the affective state, as measured by psychological tests, were recorded at four equally spaced intervals during waking hours in 25 patients with severe migraine. A dexamethasone suppression test was also performed. The results were compared with eight normal individuals studied in the same fashion.

\section{Subjects and methods}

Control subjects were paid, volunteer, young adults (ages 22-26 years) with normal wake-sleep cycles, taking no medication, and free from illness and headache. They remained in bed and on the same diet and regimen as the patients. Twenty-five patients with a history of severe recurrent migraine were recruited from patients attending the Kansas University Medical Center headache clinic. Migraine was diagnosed according to the criteria of the Ad Hoc Committee on Classification of Headache of the National Institute of Neurological Disease and Blindness (Ad Hoc Committee on Classification of Headache, 1968). None of the patients was judged as having overt psychiatric disease, notably depression. The experimental nature of the study was explained to the patients.

Patients were required to be completely free of all hormone, sedative, tranquilliser, or other medication for at least two weeks before the study. 
Patients were admitted to hospital on a Friday afternoon and placed on bed rest with bathroom privileges. A heparin lock intravenous line was placed in each patient's forearm to facilitate and minimise the pain of taking blood samples. Beginning on Saturday morning, blood samples were drawn at $0800,1200,1600$, and 2000 on Saturday and Sunday. Serum cortisol was determined by the competitive protein-binding radioligand assay (Murphy, 1967). The normal ranges are 10-20 $\mu \mathrm{g} /$ $\mathrm{dl}$ in the morning and $5-10 \mu \mathrm{g} / \mathrm{dl}$ in the afternoon. Reliability of the assay for morning cortisol values is $\pm 1.2 \mu \mathrm{g} / \mathrm{dl}$. At 2200 on Sunday, $1 \mathrm{mg}$ dexamethasone was given orally, and the final sample was drawn at 0800 on Monday. Normal response is considered to be less than $5 \mu \mathrm{g} / \mathrm{dl}$ (Nugent et al., 1965).

All patients were given the Minnesota Multiphasic Personality Inventory (MMPI) test on Friday afternoon. At the time of each blood sampling, the patient filled in a brief question form designed to sample his "mood" at the time. The short form was composed of items from the depression (D) and the psychasthenia (PT) scales of the MMPI test. Also, at the time of blood sampling, each patient recorded in a bedside diary the presence or absence of headache, and if headache were present, whether it was mild or severe. When medication was required for headache, diphenhydramine or prochlorperazine was used, since it is known that analgesics can alter blood cortisol levels (Egdahl and Richards, 1956).

Eight volunteer control subjects of comparable age range were selected by virtue of having been free of headaches and in good health, and they were placed at bed rest; plasma cortisol was sampled at the same times as the patients. Psychological testing was performed and dexamethasone test was also given as for patients.

\section{Results}

DIURNAL VARIATIONS IN CORTISOL LEVEL

To confirm the diurnal variation of plasma cortisol levels in these patients an analysis of variance for repeated measures with two-way repeats (in this case four time periods in two days) was performed. The analysis showed that the observed pattern of changes throughout the day was significant at the 0.001 level. Any differences between days was not significant $(P=0.51)$. The observation of the single day 3 plasma cortisol was not included in this analysis since it reflected suppression of plasma cortisol levels by the use of dexamethasone.

All eight normal control subjects also showed the expected circadian rhythm of plasma cortisol levels with a steady fall in the level from 0800 to 2000 on both days. The variation among the normal subjects was not great, standard deviations ranging from $1.89 \mu \mathrm{g} / \mathrm{dl}$ at 2000 on day 2 , to $5.39 \mu \mathrm{g} / \mathrm{dl}$ at 1200 , on day 1 . All control subjects showed the expected suppression of plasma cortisol to less than $5 \mu \mathrm{g} / \mathrm{dl}$ in the morning sample 10 hours after the $1 \mathrm{mg}$ dose dexamethasone.

DIFFERENCES IN PLASMA CORTISOL LEVELS BETWEEN PATIENTS AND CONTROL SUBJECTS

Mean plasma cortisol values for migraine patients were higher than those of control subjects for most hours tested except for the 2000 on day 2 , when controls were slightly higher. Using the repeated measures technique, overall significance between migraine and control subjects could not be shown $(P=0.24)$. When the time periods were studied independently, however, several important differences between migraine and control groups emerged. The amount of variation in the migraine group was striking. Standard deviations for the patient group were higher than those for the control subjects at every time period. At four of the eight time periods during the first two days, thise difference in variation was statistically significant. Probability levels for the obtained $F$ values rangedo from 0.001 to 0.03 (Table 1). This fact helped too explain the difficulty of obtaining significance foro the difference between the groups in the repeated measures analysis. In spite of the large variation, however, we were able to show significant differences between control subjects and patients at two time periods, the 2000 readings on both day 1 and day 2 ( $P$ values 0.01 and 0.02 respectively) as shown in Table 1.

Because of the great similarity among the control subjects (as evidenced by low standard deviations) in contrast to the large disparity among the patient group, we suspected that subgroups

Table 1 Mean cortisol values at four hourly intervals for two days in 25 migraine patients and eight control subjects

\begin{tabular}{|c|c|c|c|c|c|}
\hline & \multirow[t]{2}{*}{ Time } & \multicolumn{2}{|c|}{ Mean value $(\mu g / d l)$} & \multicolumn{2}{|c|}{ Standard deviation } \\
\hline & & Patient & Control & Patient & Control \\
\hline Day 1 & $\begin{array}{l}0800 \\
1200 \\
1600 \\
2000\end{array}$ & $\begin{array}{r}16.50 \\
10.70 \\
7.50 \\
5.31\end{array}$ & $\begin{array}{r}14.60 \\
7.00 \\
5.30 \\
1.59\end{array}$ & $\begin{array}{l}7.57 \\
9.00 \\
5.72 \\
5.99\end{array}$ & $\begin{array}{l}4.71 \\
5.39 \\
4.27 \\
2.16^{*}\end{array}$ \\
\hline Day 2 & $\begin{array}{l}0800 \\
1200 \\
1600 \\
2000\end{array}$ & $\begin{array}{r}14.84 \\
11.66 \\
6.60 \\
6.59\end{array}$ & $\begin{array}{r}16.44 \\
7.08 \\
6.26 \\
2.85\end{array}$ & $\begin{array}{l}9.55 \\
9.15 \\
5.73 \\
6.70\end{array}$ & $\begin{array}{l}4.03^{*} \\
4.14^{*} \\
3.89 \\
1.89^{*}\end{array}$ \\
\hline
\end{tabular}

*Variances significantly different at $\mathbf{0 . 0 5}$ level. 
among the patients might be causing the great variation. Thus, a "standard" graph was created showing in a shaded area one standard deviation above and below the mean for plasma cortisol values of control subjects over the time period of the study. Figure 1 shows this range of values in control subjects (shaded area) with the superimposed curve of mean values for the 25 migraine patients. Each individual patient's levels of plasma cortisol were then superimposed on the standard graph and four patterns (later combined to two groups, see below) emerged as follows. Seven of the 25 patients had plasma cortisol values that were consistently higher than the shaded area (one standard deviation above the mean) throughout the time period. A second group of seven cases was best characterised as having an aberrant pattern with respect to their plasma cortisol levels throughout the day. The most common abnormality in these aberrant cases was a rise in plasma cortisol at a time when all control subjects and most patients were showing a fall. The third identifiable subgroup (five cases) evidenced low plasma cortisol values at many time periods throughout two days. The remaining six cases showed patterns essentially similar to those of control subjects.

Since all studies of the association of hypothalamic-pituitary-adrenal (HPA) abnormalities with pain or psychiatric disease or both have demonstrated only hyperfunction as evidenced by elevated plasma cortisol, we decided to consider the low plasma cortisol pattern to be a probable

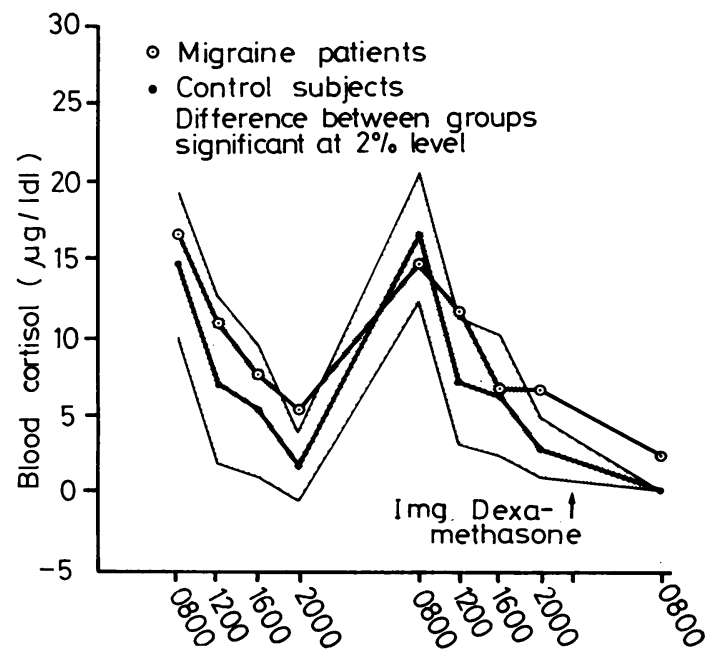

Fig. 1 Mean cortisol levels for 25 migraine patients compared with mean for 10 normal subjects $( \pm 1$ standard deviation). variant of normal and these individuals (normal and low plasma cortisol values) will subsequently be referred to as group B. The aberrant pattern, (two examples of which are shown in Figs. 2 and 3 ), we considered a probable variant of the consistently high group (two examples of which are seen in Figs. 4 and 5). The latter two groups will be referred to collectively as group A.

RELATIONSHIP OF PLASMA CORTISOL TO PAIN

To answer the question whether the elevated levels of plasma cortisol of some migraine patients might

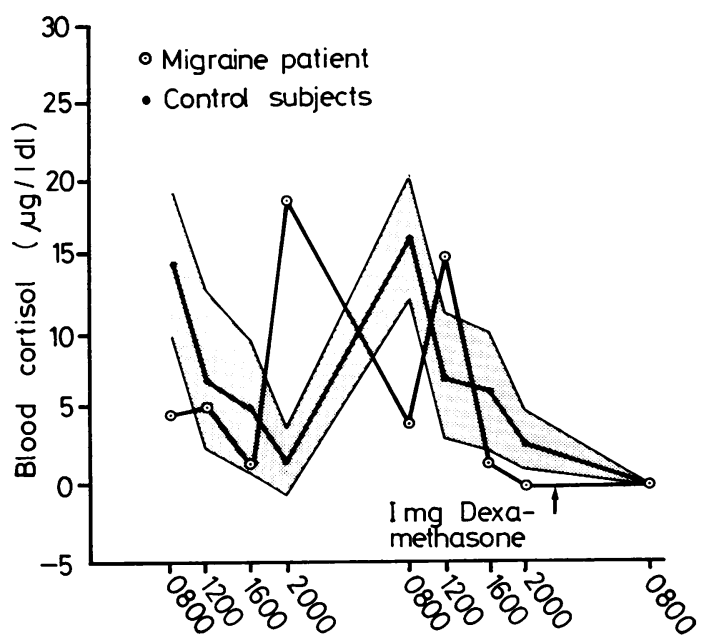

Fig. 2 Aberrant diurnal blood cortisol rhythm in female migraine patient aged 37 years compared with values for control subjects.

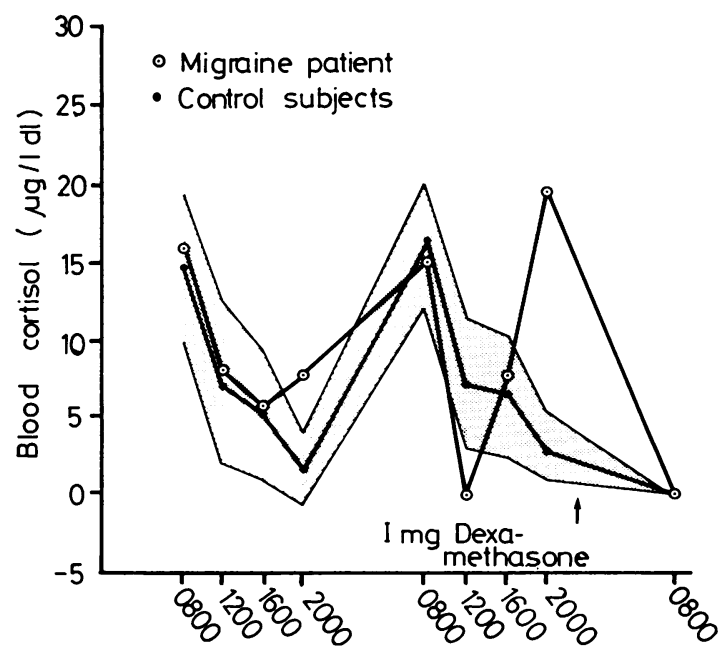

Fig. 3 Aberrant diurnal blood cortisol pattern in male migraine patient aged 41 years compared with values for control subjects. 


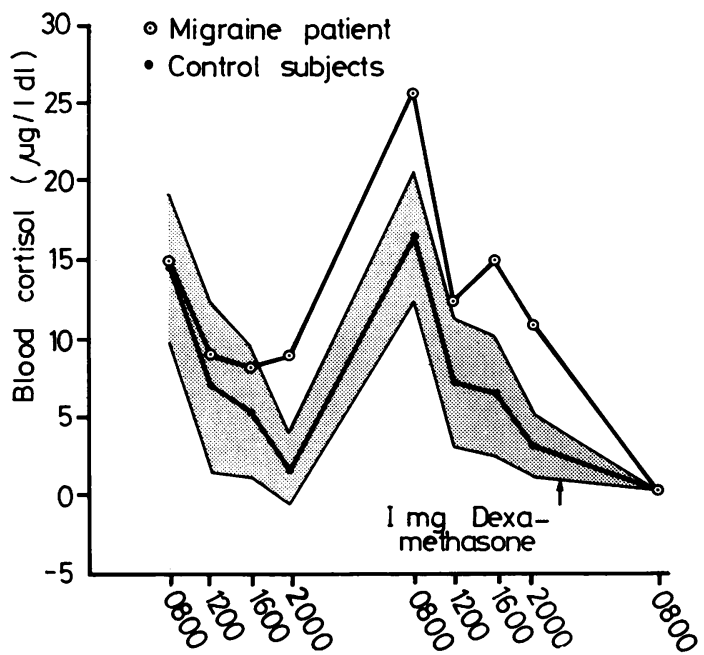

Fig. 4 Consistently high blood cortisol values in male migraine patient aged 25 years compared with values for control subjects.

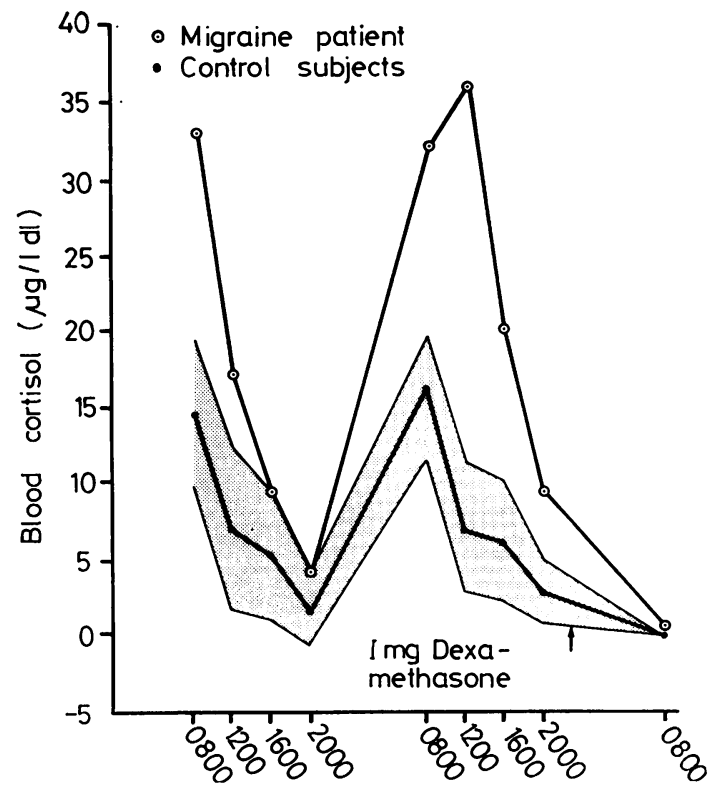

Fig. 5 Consistently high blood cortisol values in female migraine patient aged 21 years compared with values for control subjects.

be the result of increased pain itself, the association between pain and plasma cortisol level was examined. Because of the demonstrated diurnal variations of plasma cortisol, it was necessary to adjust observed levels to account for the time of day. To accomplish this, $Z$ scores were computed -this involves subtracting from each patient's observed plasma cortisol level the mean level of plasma cortisol obtained from the control group at that time period, and dividing by the corresponding standard deviation. The scores obtained could thus be interpreted as representing by how many standard deviations above or below the expected plasma cortisol level of the time period the subject's cortisol level had risen or fallen. Pain scores were constructed by assigning the value of zero to no headache, 1 to mild headache, and 2 to severe headache pain. Correlation coefficients were computed in two ways: firstly by using all categories of pain and correlating pain score with the plasma cortisol $\mathrm{Z}$ score and using the Pearson product-moment correlation coefficient; secondly, by dividing patients into those who did and those who did not have severe headache, and correlating, using the point-biserial correlation coefficient with the same $\mathrm{Z}$ score. The obtained Pearson correlation coefficients are shown in Table 2. While the correlations are quite varied and most are not statistically significant from zero, the overall association of pain with elevated plasma cortisol is definitely positive. In fact, it can be shown that, in the absence of true correlation, the्ष $\nexists$ chance of obtaining the two distributions of cors relation coefficients mentioned, the first of which is shown in Table 2 , is extremely small $(P<0.0 \mathbb{Q}$ and $\mathbf{P}<0.01$ respectively), using the Wilcoxor signed rank test for the significance of the differbe ence of the median from zero.

Looking at the association between pain an level of plasma cortisol over all time periods, we divided the time periods into three groups, one in which the patients had no pain, one in which only mild headache pain was noted, and one in which severe pain was present. The average plasma cortisol $\mathrm{Z}$ scores were then computed for each pain group. Once again, a trend was noted, with mean $\mathrm{Z}$ scores for the three groups of 0.053 ,

Table 2 Correlation coefficients for association between pain score and standardised plasma cortisol score ( $Z$ score) by time of day for 25 migraine patients

\begin{tabular}{cc}
\hline Time of day & $\begin{array}{c}\text { Correlation } \\
\text { coefficient } \\
r\end{array}$ \\
\hline 0800 & 0.1615 \\
1200 & 0.2551 \\
1600 & 0.3285 \\
2000 & -0.0692 \\
0800 & 0.3577 \\
1200 & 0.1869 \\
1600 & 0.2541 \\
2000 & 0.2625
\end{tabular}

Wilcoxon signed rank test for median $r$ different from zero, significant at $2 \%$ level. 
0.641 , and 1.526 respectively, indicating that in time periods where severe pain was recorded, plasma cortisol levels averaged out to about 1.5 standard deviations above the normal control means for those time periods. Since the time periods could not be considered independent observations, tests of significance could not be done, but the results are quoted because of the importance of possible clinical implications.

Examinations of the $\mathrm{Z}$ score distributions within pain group time periods revealed that while there was a tendency for high levels of plasma cortisol to occur when pain was present, there were so many exceptions that the presence of headache could not be the sole explanation for these high levels. On many occasions, severe pain was reported at the time of normal or low values of plasma cortisol, and, conversely, high levels occurred when pain was absent. For example, in patient ED (Fig. 5) severe pain was reported at 2000 on day 1 , when the plasma cortisol level was close to normal range, while mild pain was reported at 0800 on day 2 when it was extremely high. Patient JA (Fig. 4) with consistently high levels of plasma cortisol, reported no headache at any time during the study. Conversely, JG (Fig. 3) did report his only severe headache at the time of a markedly raised level of plasma cortisol.

Six patients failed in varying degrees to suppress plasma cortisol levels in response to the test dose of dexamethasone. Four of these patients fell into the group of consistently high plasma cortisol, the other two into the aberrant group. The entire study was repeated on two of these patients, and in this second study one patient failed completely to suppress plasma cortisol in response to dexamethasone, the late evening cortisol value immediately before dexamethasone being $8.4 \mu \mathrm{g} / \mathrm{dl}$ and the 0800 value 12 hours later being $20.9 \mu \mathrm{g} / \mathrm{dl}$. The second patient on whom this study was repeated again showed high values on both days and lack of response to dexamethasone.

RELATIONSHIP OF PLASMA CORTISOL LEVEL TO PSYCHOLOGICAL TEST RESULTS

The MMPI test results were divided into normal and abnormal: the latter was defined as any individual scoring 70 or more $\mathrm{T}$ score on one or more of the 10 clinical scales. Test results on all control subjects were within normal limits. In the patient group, eight group B patients (normal plasma cortisol levels) had normal MMPI results and the remaining three had slight increases only in the "somatic" scale. Of the 14 group A patients (elevated or aberrant plasma cortisol levels), seven had abnormal MMPI profiles (50\%). Six of these had three or more scales scoring above 70 . The remaining patient showed a slight increase on two somatic scales ( $\mathrm{Hs}$ and $\mathrm{Hy}$ ). In the six subjects with elevation of three or more scales, depression was one of these scales in four subjects. In no control subject or any other patient was an elevation of $D$ scale found. Of particular note is the negative finding that in seven of the group A patients there was no evidence of abnormality on the MMPI test.

The D and PT scales of the short psychological tests administered at time of blood sampling were then studied for their possible association with plasma cortisol values because of the above findings. On these short forms, as on the initial MMPI, we found a significant difference between the mean scores of total patient group and controls, using repeated measures analysis of variance described above, both for $\mathrm{D}(\mathrm{P}=0.005)$ and $\mathrm{PT}$ scores $(P=0.02)$. No evidence of diurnal variation was found in either measurement.

Because of the findings on the initial MMPIs and the known association of depression with elevated plasma cortisol, it was decided to study the $D$ scale levels of the two originally described patient groups, A and B, even though, as noted above, elevations of $\mathrm{D}$ scale to abnormal levels were not found in any group $B$ patients, and in only four of 14 group A patients. Although the difference between the mean $D$ scales of group $A$ and group B patients was not statistically significant, possibly because of small numbers and the great variation, there is a consistent tendency for the group with abnormal plasma cortisol levels to have higher $\mathrm{D}$ scores.

The possibility that abnormal plasma cortisol values in patients with normal MMPIs were attributable to severe pain was next studied. On only three out of the seven group A patients with normal MMPIs was severe pain reported at any time during the study.

\section{Discussion}

The most striking findings in this study are the large variation in plasma cortisol values in migraine patients as compared to control subjects, and the occurrence of two patterns of abnormal circadian levels of plasma cortisol occurring in subgroups of migraine patients - one of consistently high levels of plasma cortisol and another of one or more aberrant high values occurring during the day.

Although the mean plasma cortisol values for 
the migraine patients were higher than those of control subjects at most times tested, the difference was not significant, but the variation in the migraine group was greater than that of controls at all time periods and significantly greater at four of the eight time periods tested. These findings suggest a similarity to those of Krieger and Krieger (1966) who studied circadian changes in plasma cortisol levels in a series of subjects with disease of the temporal lobe, pretectum, or hypothalamus, and two control series-one with central nervous system disease outside these areas and another with no disease. They found no significant difference in average plasma cortisol levels between the three groups. The study group, however, differed significantly from both control groups in the extent of variation of plasma cortisol from the diurnal slope of the linear trend.

The second major finding, that of elevated plasma cortisol occurring either consistently or at occasional sampling times, could have been the result of pain or of the presence of some type of psychological or emotional state. Shenkin (1964) found that of 66 patients with pain that he considered objective (acute lumbosacral strain, herniated lumbar disc, tic douloureux, and so on), $86.4 \%$ had some abnormality of diurnal values of plasma cortisol. The abnormality was always one or more high values. By contrast, 45 patients judged to have psychogenic pain (low back pain, tension headache) were found to have normal mean and diurnal patterns of plasma cortisol in $84.4 \%$ of the cases. Lascelles et al. (1974), studying the same problem in 25 subjects, found mean level of plasma cortisol elevated over controls in both patients with psychogenic and those with organic pain. The plasma cortisol levels, however, showed greater elevation in the latter group. The normal diurnal variation was also reduced in both pain groups, again more in the "organic group."

Migraine presents two particular problems in the study of pain-firstly, the determination as to whether any specific headache is "organic" in origin is particularly difficult, and secondly, the pain is characteristically episodic. Pain may or may not be present at the time of sampling plasma cortisol. We shall not enter into the question of the organic nature of pain in our patients. There is, of course, massive evidence for physiopathological abnormalities occurring during migraine attacks, and the organic-psychogenic dichotomy seems an inapplicable oversimplification in this problem.

The question of whether plasma cortisol values were elevated specifically at times of pain was ad- dressed by computation of $\mathbf{Z}$ scores described above. We found that there was an overall correlation of pain with elevation of plasma cortisol. In time periods where severe pain was recorded, it was found that plasma cortisol levels averaged out to about 1.5 standard deviations above the normal control means for those time periods. Pain per se is, then, probably one factor contributing to the abnormal levels of plasma cortisol in some of these patients. Whether pain can be separated from "psychological stress" is dubious, and it is well known that various strong environmental stimuli producing suspense and anxiety can increase plasma cortisol (Mason, 1968; Norman and Turter, 1968; Charters et al., 1969; Brown and Heninger, 1975). We found, however, no significant diurnal variation in our psychological tests administered at the times of plasma cortisol testing. It is doubtful, therefore, whether transient psychological stress (at least as here tested) is responsible for the increase in plasma cortisol. Sacher (1973) has reviewed the evidence from several studies on populations under a variety of transient stressful situations that the adrenal-cortical response to such stress is "relatively slight" and very inconstant Furthermore, it has been noted by Smith (1973) that "no parameter of corticoid response sensitivelyo reflects different intensities of physical stimuli of emotional excitement between threshold and maximal emotional stimulation." That pain cannot bo the sole cause of the elevation of plasma cortisol is evident, not only from statistical evidence but from the many times when severe pain was reported with normal plasma cortisol levels and conversely when elevated plasma cortisol occurred in the absence of pain.

The inconstant association between plasma cortisol and pain is, of course, consistent with the many observations that somatic "stress" in the absence of pain may cause marked increases in plasma cortisol, notably in anaesthetised patients undergoing surgery (Charters et al., 1969; George et al., 1974).

It is well known that plasma cortisol is increased in depressive states, and it is most consistently the psychotic depression that has been associated with this abnormality (Carroll, 1976; Carroll et al., 1976a, b). As to other psychiatric conditions, marked deviations from normal plasma cortisol levels have been reported in anorexia nervosa and in schizophrenia (Suwa et al., 1974; Katz et al., 1976), although the latter finding has been challenged (Carroll, 1976). In our study, all control subjects and eight of 11 migraine patients with normal plasma cortisol levels had normal MMPI 
test results, with minimal abnormality in the other three. In no control subject or patient with normal plasma cortisol, specifically, was there an increase in the depression (D) scale. Conversely, of the 14 patients with high plasma cortisol level or abnormal diurnal variation, seven had abnormal MMPI scales. The D scale was raised in four of these seven; and in six, two or more other scales were elevated. In none of these cases, all of whom had been carefully followed in a headache clinic for several months, had a clinical diagnosis of depression or other overt psychiatric disease been made.

The relationship between depression and migraine is complex. The most well-documented facet of the problem is the fact that amitriptyline, a tricyclic antidepressant, is an effective prophylactic drug against migraine in a part of the patient population (Couch and Hassanein, 1976). The results of the present study provide evidence for another possible link between depression and migraine, and suggest that one factor in the elevation of plasma cortisol in some of these cases may be unsuspected depression. The association of depression with raised plasma cortisol has been amply documented (Carroll, 1976). It is of interest, however, that in none of our patients had clinical depression been diagnosed, and in three individuals with abnormal levels of plasma cortisol the MMPI test did not show elevation of the D scale. Furthermore, none of the abnormal MMPIs were characteristic of severe depression but reflected in addition anxiety, social alienation, bodily preoccupation, and lack of deep interests. It is improbable, therefore, that the existence of overt depression in these cases is the sole explanation for the elevation of plasma cortisol. This finding is of particular interest because hypothalamopituitary-adrenal (HPA) dysfunction has previously been described consistently only in severe depression, and has been found correlated more with somatic than with psychological features. To our knowledge, other studies of HPA function in less severe emotional disturbance with somatic symptoms (except for pain studies cited) have not been done.

Failure of suppression of plasma cortisol by dexamethasone has been found regularly in populations with endogenous depression and has not been found with other psychiatric disease (Carroll et al., 1976b). In the present study, six individuals did not show normal suppression to dexamethasone. Two of these patients had an elevated D score on MMPI scale, four did not. Of the latter four, three had completely normal MMPI scores, on an elevated somatic scale. Of our four patients with elevated D scores on MMPI, two showed normal suppression with dexamethasone, two did not. In summary, the dexamethasone test also showed abnormality in some of the migraine patients, not consistently associated with depression. We did not test plasma cortisol beyond the 12 hour sample and, therefore, do not know whether these patients who did suppress might have shown an early "escape" as has been described.

We are aware of the fact that the circadian rhythm of cortisol secretion is comprised of a series of episodic pulses, as shown by several investigators (Weitzman et al., 1966, 1971; Hellman et al., 1970). These pulses are normally related inconstantly to environmental stimuli including several anxiety producing events. It has, however, been demonstrated that specific environmental stimuli, presumably extremely stressful, such as preoperative preparation, can produce major secretory surges of cortisol, which can clearly be detected as producing alterations in the circadian rhythm (Czeisler et al., 1976). Our results show a clear distinction between control subjects where there was a total absence of such surges, and the considerable group of patients where the circadian rhythm was clearly distorted. This disparity cannot be ascribed to any procedures or environmental stimuli that were different for patients and control subjects during the study period, since all subjects were treated identically.

It seems, in summary, that the abnormal circadian rhythms of plasma cortisol seen in over half of our migraine patients had some, but inconstant, association with pain. A similar statement could be made about the association with depression. We postulate that the abnormal plasma cortisol circadian rhythms occurring in some migraine patients may be the result of a complex interaction of these two aetiological factors. There is, however, also the possibility that the HPA abnormality in some cases of migraine is a reflection of primary hypothalamic dysfunction of unknown mechanism as reported by Krieger and Krieger (1966) in their study of organic brain disease. In this case, of course, the pain and psychological findings might well be secondary phenomena. Finally, the occurrence of a group of migraine patients with normal circadian rhythms of plasma cortisol associated with normal MMPI tests gives strong evidence of the heterogeneity of the population defined as migrainous and the desirability of pursuing the study of the neuroendocrine status of this interesting group of patients. 
This work was supported by the Martha Jane Starr Fund and the Elmer F. Pierson Fund of the Kansas University Endowment Association and NIH Grant No RR 828.

\section{References}

Ad Hoc Committee on Classification of Headache of National Institute of Neurological Diseases and Blindness (1968). In Handbook of Clinical Neurology, vol. 5. Edited by P. J. Vinken and G. W. Bruyn. North-Holland: Amsterdam.

Brown, W. A., and Heninger, G. (1975). Cortisol, growth hormone, free fatty acids, and experimentally evoked affective arousal. American Journal of Psychiatry, 132, 1172-1176.

Carroll, B. J. (1976). Limbic system-adrenal cortex regulation in depression and schizophrenia. Psychosomatic Medicine, 38, 106-121.

Carroll, B. J., Curtis, G. C., and Mendels, J. (1976a). Neuroendocrine regulation in depression. I. Limbic system-adrenocortical dysfunction. Archives of General Psychiatry, 33, 1039-1044.

Carroll, B. J., Curtis, G. C., and Mendels, J. (1976b). Neuroendocrine regulation in depression. II. Discrimination of depressed from nondepressed patients. Archives of General Psychiatry, 33, 1051-1057.

Charters, A. C., Odell, W. D., and Thompson, J. C. (1969). Anterior pituitary function during surgical stress and convalescence. Radioimmunoassay measurement of blood TSH, LH, FSH and growth hormone. Journal of Clinical Endocrinology and Metabolism, 29, 63-71.

Couch, J. R., and Hassanein, R. S. (1976). Migraine and depression: Effect of amitriptyline prophylaxis. Transactions of the American Neurological Association, 101, 1-4.

Czeisler, C. A., Ede, M. C. M., Regestein, Q. R., Kisch, E. S., Fang, V. S. and Ehrlich, E. N. (1976). Episodic 24hour cortisol secretory patterns in patients awaiting elective cardiac surgery. Journal of Clinical Endocrinology and Metabolism, 42, 273-283.

Egdahl, R. H., and Richards, J. B. (1956). Effect of chlorpromazine on pituitary ACTH secretion in the dog. American Journal of Physiology, 185, 235-238.

George, J. M., Reier, C. E., Lanese, R. R., and Rowen, J. M. (1974). Morphine anesthesia blocks cortisol and growth hormone response to surgical stress in humans. Journal of Clinical Endocrinology and Metabolism, 38, 736-741.

Hellman, L., Nakada, F., Curti, J., Weitzman, E. D., Kream, J., Roffwarg, H., Ellman, S., Fukushima, D. K., and Gallagher T. F. (1970). Cortisol is secreted episodically in normal man. Journal of Clinical Endocrinology and Metabolism, 30, 411.

Katz, J. L., Boyar, R. M., Weiner, H., Gorzynski, G., Roffwarg H., and Hellman, L. (1976). Toward an elucidation of the psychoendocrinology of anorexia nervosa. In Hormones, Behavior and Psychopathology, pp. 263-283. Edited by E. J. Sachar. Raven Press: New York.
Krieger, D. T., and Krieger, H. P. (1966). Circadian

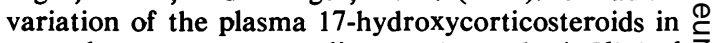
central nervous system disease. Journal of Clinical Endocrinology and Metabolism, 26, 929-940.

Lascelles, P. T., Evans, P. R., Merskey, H., and $\widehat{0}$ Sabur, M. A. (1974). Plasma cortisol in psychiatric and neurological patients with pain. Brain, 97, 533538.

Mason, J. W. (1968). A review of psychoendocrine research on the pituitary-adrenal cortical system. Psychosomatic Medicine, 30, 576-607.

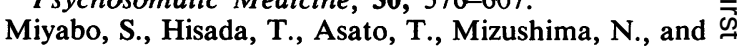
Ueno, K. (1976). Growth hormone and cortisol $\frac{C}{8}$ responses to psychological stress: comparison of normal and neurotic subjects. Journal of Clinical $\frac{\text { क }}{\circ}$ Endocrinology and Metabolism, 42, 1158-1162.

Murphy, B. E. (1967). Some studies of the proteinbinding of steroids and their application to the $\infty$ routine micro and ultramicro measurement of $\overrightarrow{0}$ various steroids in body fluids by competitive protein-binding radioassay. Journal of Clinical Endo- $\vec{\omega}$ crinology and Metabolism, 27, 973-990.

Norman, N., and Turter, A. R. (1968). Radioimmunoassay studies with human growth hormone and a pituitary lipid mobilizing factor. Acta Endocrino- $\mathrm{N}$ logica, 58, 318-338.

Nugent, C. A., Nichols, T., and Tyler, F. H. (1965\$ Diagnosis of Cushing's syndrome. Single dose dexa methasone test. Archives of Internal Medicine, 11 웅 172-176.

Sachar, E. J. (1973). Psychological factors relating activation and inhibition of the adrenocortical stress response in man: a review. Progress in Brain R search, 32, 316-324.

Shenkin, H. A. (1964). The effect of pain on the diurnal pattern of plasma corticoid levels. Neurology (Minneapolis), 14, 1112-1117.

Smith, G. P. (1973). Adrenal hormones and emotional behavior. In Progress in Physiological Psychology, vol. 5, pp. 299-351. Edited by E. Stellar and J. M. Sprague. Academic Press: New York.

Suwa, N., Yamashita, I., Moroji, T., Yamazaki, K., Okada, F., Saito, Y., Asano, Y., and Fujieda, T. $\overrightarrow{\bar{O}}$ (1974). Circadian rhythm of plasma cortisol in 3 endogenous psychoses. In Psychoneuroendocrinology. Workshop Conference of the International Society for Psychoneuroendocrinology, Mieken, 1973. Edited by N. Hatotani. S. Karger: Basel.

Weitzman, E. D., Fukushima, D., Nogeire, C., Roffwarg, H., Gallagher, T. F., and Hellman, L. (1971). Twenty-four hour pattern of the episodic secretion of cortisol in normal subjects. Journal of Clinical Endocrinology and Metabolism, 33, 14.

Weitzman, E. D., Schaumberg, H., and Fishbein, W. (1956). Plasma 17-hydroxycorticosteroid levels during sleep in man. Journal of Clinical Endocrinology and Metabolism, 26, 121.

Yates, F. E., and Urquhart, J. (1962). Control of N plasma concentrations of adrenocortical hormones. Physiological Reviews, 42, 359-443. 